\title{
Wolbachia Biocontrol Strategies for Arboviral Diseases and the Potential Influence of Resident Wolbachia Strains in Mosquitoes
}

\author{
Claire L. Jeffries $^{1}$ - Thomas Walker ${ }^{1}$ \\ Published online: 2 February 2016 \\ (C) The Author(s) 2016. This article is published with open access at Springerlink.com
}

\begin{abstract}
Arboviruses transmitted by mosquitoes are a major cause of human disease worldwide. The absence of vaccines and effective vector control strategies has resulted in the need for novel mosquito control strategies. The endosymbiotic bacterium Wolbachia has been proposed to form the basis for an effective mosquito biocontrol strategy. Resident strains of Wolbachia inhibit viral replication in Drosophila fruit flies and induce a reproductive phenotype known as cytoplasmic incompatibility that allows rapid invasion of insect populations. Transinfection of Wolbachia strains into the principle mosquito vector of dengue virus, Stegomyia aegypti, has resulted in dengue-refractory mosquito lines with minimal effects on mosquito fitness. Wolbachia strains have now been established in wild St. aegypti populations through open releases in dengue-endemic countries. In this review, we outline the current state of Wolbachia-based biocontrol strategies for dengue and discuss the potential impact of resident Wolbachia strains for additional target mosquito species that transmit arboviruses.
\end{abstract}

Keywords Mosquitoes $\cdot$ Arboviruses $\cdot$ Mosquito biocontrol · Wolbachia bacteria

This article is part of the Topical Collection on Viral Tropical Medicine

Thomas Walker

thomas.walker@1shtm.ac.uk

Claire L. Jeffries

claire.jeffries@1shtm.ac.uk

1 Department of Disease Control, Faculty of Infectious Diseases, London School of Hygiene and Tropical Medicine, Keppel Street, London WC1E 7HT, UK

\section{Introduction}

Arboviruses that cause human disease are predominantly transmitted by mosquitoes. Although there are more than 80 different arboviruses, most human cases result from infection with dengue virus (DENV) and other closely related Flaviviruses. The genus Flavivirus also includes West Nile virus (WNV), Yellow fever virus (YFV), Zika virus (ZIKV) and Japanese encephalitis virus (JEV). These medically important arboviruses are transmitted by several species of Culicine mosquitoes (Table 1). It is estimated that $40 \%$ of the world's population live in areas at risk for DENV infection in more than 100 countries. Global DENV infections range from 100-390 million per year, with 100 million symptomatic infections leading to 12,500 deaths per year $[1 \bullet$. Dengue is an epidemic disease that occurs in tropical areas of Southeast Asia and South America and has a significant impact on developing countries [2]. Rare cases have been documented in the USA and southern Europe, and dengue is 're-emerging' mostly due to the expansion of the geographical range of the principal mosquito vector, Stegomyia (St.) (Aedes) aegypti, through globalization and climate change [3]. There are currently no effective vaccines for DENV, so supportive treatment is the only method to reduce morbidity or prevent mortality for infected patients. Prevention of DENV transmission relies on mosquito vector control but this has not seen much success in recent years. Attempts to reduce the larval breeding of St. aegypti, predominantly an urban species, over a large geographical area have been difficult to achieve. There is also a delay between implementing larval control and achieving an impact on the adult mosquito population already transmitting disease. When dengue epidemics occur, the usual response is to use outdoor space spraying of insecticides. Although this targets the adult mosquito, insecticide resistance has been problematic in many countries. The options for developing 
Table 1 Culicine mosquito vectors, arboviruses they transmit at significant levels in field populations and natural resident Wolbachia strains

\begin{tabular}{|c|c|c|}
\hline Mosquito species & $\begin{array}{l}\text { Arbovirus } \\
\text { transmission }\end{array}$ & $\begin{array}{l}\text { Resident } \\
\text { Wolbachia } \\
\text { strain }\end{array}$ \\
\hline Stegomyia aegypti & $\begin{array}{l}\text { DENV, YFV, } \\
\text { CHIKV ZIKV }\end{array}$ & - \\
\hline Stegomyia albopicta & DENV, CHIKV & $w \mathrm{AlbA}, w \mathrm{AlbB}$ \\
\hline Stegomyia africana & YFV & - \\
\hline Stegomyia simpsoni & YFV & - \\
\hline Aedes bromeliae & YFV & Unnamed \\
\hline Aedes fluviatilis & YFV experimentally & $w$ Flu \\
\hline Culex tritaeniorhynchus & JEV, RVFV, WNV & - \\
\hline Culex quinquefasciatus & WNV, RVFV, SLEV & $w$ Pip \\
\hline Culex pipiens & WNV, RVFV, SLEV & $w$ Pip \\
\hline Culex tarsalis & WNV, WEEV & - \\
\hline Culex univittatus & WNV, SINDV & - \\
\hline Culex theileri & RVFV & - \\
\hline Culex annulirostris & MVEV, JEV, RRV & - \\
\hline Culex nigripalpus & SLEV & - \\
\hline Culiseta morsitans & EEEV & - \\
\hline Culiseta melanura & WEEV, EEEV, WNV & - \\
\hline Coquillettidia perturbans & WNV, EEEV & Unnamed \\
\hline Haemagogus spegazzinii & YFV & - \\
\hline Ochlerotatus sollicitans & WNV, EEEV & - \\
\hline Ochlerotatus taeniorhynchus & WNV, EEEV, VEEV & - \\
\hline Ochlerotatus serratus & VEEV & - \\
\hline Ochlerotatus vigilax & RRV & - \\
\hline Ochlerotatus dorsalis & WNV, CEV & - \\
\hline Ochlerotatus melanimon & WNV, CEV & - \\
\hline Ochlerotatus triseriatus & LCV & - \\
\hline Psorophora confinnis & VEEV & \\
\hline Mansonia titillans & VEEV & Unnamed \\
\hline
\end{tabular}

$D E N V$ dengue virus, $Y F V$ yellow fever virus, $C H I K V$ chikungunya virus, $Z I K V$ zika virus, $W N V$ West Nile virus, EEEV Eastern equine encephalitis virus, JEV Japanese encephalitis virus, SLEV St Louis encephalitis virus, $R V F V$ Rift Valley fever virus, WEEV Western equine encephalitis virus, SINDV Sindbis virus, MVEV Murray Valley encephalitis virus, VEEV Venezuelan equine encephalitis virus, RRV Ross River virus, $C E V$ California encephalitis virus, $L C V$ La Crosse virus

new mosquito control strategies can be classified as either suppression (reduce or eliminate the wild mosquito population) or replacement with mosquitoes that are unable to transmit disease. A recent novel approach is the use of endosymbiotic Wolbachia bacteria to prevent DENV from replicating within the mosquito. In recent years, Wolbachia-based biocontrol has emerged as a very promising method that is environmentally friendly, safe to humans and potentially cost effective [4]. The 'eliminate dengue' project (www. eliminatedengue.com) has shown that Wolbachia can prevent DENV transmission in mosquitoes without significant fitness costs.

\section{Natural Wolbachia Infections in Mosquitoes}

Wolbachia is a genus of obligate intracellular alphaproteobacteria within the family Rickettsiaceae [5]. Wolbachia was initially found in Culex pipiens mosquitoes in 1924 [6]. Since then, many different strains of Wolbachia have been discovered in a wide range of invertebrate hosts, including many filarial nematode and arthropod species. A recent study estimated that Wolbachia naturally infects more than $65 \%$ of arthropod species [7]. Natural Wolbachia infections are present in some mosquito species that transmit human pathogens including Culex (Cx.) quinquefasciatus and Stegomyia albopicta (Aedes albopictus) (Table 1). Additional Wolbachia strains have been discovered in minor mosquito vectors of YFV including Aedes (Ae.) bromeliae [8] and Ae. fluviatilis which is infected with the $w$ Flu strain [9]. Wolbachia infections are also present in wild mosquito populations of Coquilletidia perturbans [10] and Mansonia titillans [11]. However, natural infections are absent from most Anopheles spp. (that transmit malaria), St. aegypti and Cx. tritaeniorhynchus (the major vector of JEV). Wolbachia strains are currently divided into eight $(\mathrm{A}-\mathrm{H})$ supergroups according to sequence information [5]. Wolbachia strains have the ability to manipulate host reproduction in order to enhance their own reproduction and transmission through an insect population. Wolbachia strains can induce various phenotypic effects in insects including parthenogenesis, feminization, male killing and cytoplasmic incompatibility [5].

\section{Cytoplasmic Incompatibility}

In mosquitoes, Wolbachia strains can induce cytoplasmic incompatibility $(\mathrm{CI})$, which results in the generation of unviable offspring when an uninfected female mates with a Wolbachiainfected male [5]. In contrast, Wolbachia-infected females can produce viable progeny when they mate with both infected and uninfected males, resulting in a reproductive advantage over uninfected females. The CI phenotype allows the maternally transmitted Wolbachia to efficiently invade host populations without being infectious or moving horizontally between individuals [5].

$\mathrm{CI}$ can also be the result when mating occurs between mosquitoes with two different, incompatible Wolbachia strains. This CI can occur bidirectionally if crosses of both males and females with each different Wolbachia strain are incompatible, resulting in embryonic death in both crosses. However, some crosses between mosquitoes with differing strains can instead lead to unidirectional CI, where a cross of males with strain ' $x$ ' and females with strain ' $y$ ' results in CI, whereas the reciprocal cross (males with strain $y$ and females with strain $\mathrm{x}$ ) allows the production of viable offspring. In this case, mosquitoes with Wolbachia strain $\mathrm{x}$ would have a reproductive advantage over those with strain $\mathrm{y}$ and strain $\mathrm{x}$ and 
would be predicted to successfully spread through the population, "sweeping over" strain y [12].

\section{Transinfection of Wolbachia Into Mosquitoes}

The various phenotypic effects of Wolbachia strains on natural insect hosts have led to a range of ideas on how this could be applied for mosquito-borne disease vector control. One potential strategy was investigated in the 1960s using repeated releases of Wolbachia-infected male Culex mosquitoes to suppress wild populations using CI [13]. A later discovery of the wMelPop strain of Wolbachia in Drosophila melanogaster fruit flies, which dramatically lowered the lifespan of its host [14], led to the potential use of 'life-shortening' strains to manipulate the population age structure of important mosquito vectors. Recently, Wolbachia strains from Drosophila fruit flies were found to protect their native hosts against infection by pathogenic RNA viruses $[15,16]$. Wolbachia strains are associated with a significant reduction in the viral density of a range of viruses in flies, which delays insect mortality [17].

The use of Wolbachia for mosquito biocontrol first required the stable infection (transinfection) of target mosquito species. The first target species selected was St. aegypti, the principle vector of DENV. The $w$ AlbB strain of Wolbachia was successfully established in St. aegypti using embryo cytoplasm transfer from closely related St. albopicta mosquitoes [18]. St. aegypti lines were later stably transinfected with $w$ MelPop and $w \mathrm{Mel}$ strains from the native host Drosophila melanogaster [19, 20]. These transinfected Wolbachia strains significantly reduced the vector competence of St. aegypti for DENV in laboratory experiments [20-22]. High levels of Wolbachia bacteria infected the tissues that play a crucial role in DENV replication within mosquitoes. The presence of infectious DENV in saliva was completely inhibited by Wolbachia [20].

Successful Wolbachia-based biocontrol would also require invasion of wild mosquito populations. Wolbachia-infected females must vertically transmit the bacteria to their progeny at a high frequency. All three transinfected Wolbachia strains ( $w$ AlbB, $w$ Mel and $w$ MelPop) show maternal transmission rates close to $100 \%$ and induce high levels of CI in St. aegypti [18-20]. The ability of transinfected Wolbachia to successfully invade wild St. aegypti mosquito populations will depend on a balance between negative selection imposed by fitness costs of the bacteria on the mosquito and positive selection associated with CI induction. The wMelPop strain results in significant fitness costs (including impacts on adult longevity and fecundity) so was considered inappropriate for initial test releases into wild mosquito populations.

\section{The Release of Wolbachia-Infected Mosquitoes}

The invasive potential of Wolbachia strains was first tested in a semi-field facility that simulated the natural habitat of St. aegypti in north Queensland, Australia [20]. Successful trials led to mosquitoes infected with the $w \mathrm{Mel}$ strain being released into the wild through open releases in two locations near Cairns in north Queensland, Australia [23]. The wMel strain successfully invaded the two natural populations, infecting nearly $100 \%$ of the local population within a few months following releases. Prior to the release of Wolbachia-infected mosquitoes in Australia, extensive engagement with the communities in the release areas took place to determine the attitudes and levels of knowledge about dengue and mosquitoes. The success of these initial trials has led to further releases in DENV endemic countries such as Indonesia, Vietnam and Brazil (www.eliminatedengue. com). Continued success of a release program will require maintenance of an inhibitory effect on DENV replication in wild Wolbachia-infected St. aegypti populations. Vector competence experiments carried out with field $w$ Mel-infected mosquitoes, collected 1 year following field release, indicated insignificant DENV replication and dissemination [24•]. Currently, work is being undertaken to determine the optimal Wolbachia strain for applied use that can balance the effects on pathogen transmission and fitness costs to the mosquito. Recent mathematical models of DENV transmission incorporating the dynamics of viral infection in humans and mosquitoes predict that $w \mathrm{Mel}$ would reduce the basic reproduction number, $\mathrm{R}_{0}$, of DENV transmission by approximately $70 \%$ [25]. At the current time, it remains unclear what effect Wolbachia will have on DENV transmission and dengue epidemiology in the field. A cluster-randomized trial is premature because the choice of Wolbachia strain for release and deployment strategies is still being optimized [26•].

\section{Potential Use of Wolbachia for Additional Arboviral Diseases}

Although Wolbachia-infected St. aegypti were originally generated for biocontrol of dengue, they are likely to have the added benefit of reducing transmission of additional arboviruses vectored by this mosquito species, including chikungunya virus (CHIKV) [21] and YFV [27] and potentially ZIKV. Like St. aegypti, mosquito species that are the principle vector of arboviruses and contain no natural Wolbachia infections are likely to represent the most feasible transinfection targets. JEV is predominantly transmitted by Cx. tritaeniorhynchus mosquitoes, and a Wolbachia-based biocontrol strategy has the potential to reduce transmission if stably infected lines can be generated [28]. JEV is part of the same genus as DENV (Flavivirus), so Wolbachia strains would likely provide similar inhibitory effects on transinfected mosquitoes. Drosophila Wolbachia strains grow to high densities in their native and transinfected hosts and provide strong inhibition of both insect viruses 
in Drosophila [16] and DENV in mosquitoes [20]. Successful transinfection of Drosophila Wolbachia strains in $C x$. tritaeniorhynchus and the release of stably infected mosquitoes which enable the Wolbachia strain to invade wild populations, with strong viral interference characteristics, would be likely to significantly reduce JEV transmission [28].

\section{Resident Wolbachia Strains in Mosquitoes and Effects on Arbovirus Replication}

Some major mosquito vectors of arboviral diseases harbor natural Wolbachia infections. Both DENV and CHIKV are also transmitted by St. albopicta, which contain two resident strains of Wolbachia; wAlbA and wAlbB [29]. The principle vectors of WNV in the USA, Cx. pipiens and $C x$. quinquefasciatus, contain a natural Wolbachia strain, wPip. Minor differences in vector competence may be due to the presence of these resident Wolbachia strains. For example, Cx. quinquefasciatus, infected with the wPip strain of Wolbachia, is generally less susceptible to WNV than $C x$. tarsalis [30], which is not infected with Wolbachia. However, resident Wolbachia infections in mosquitoes do not impact arboviral transmission to the same extent as transinfected Drosophila Wolbachia strains, as reviewed in [28]. One mechanism suggested for this difference is that the newly transinfected strains trigger an immune response in their new invertebrate hosts, which also has antiviral effects, whereas native Wolbachia strains have been present in the host long enough that the host no longer generates such an immune response. However, the complexity in the interaction between Wolbachia, the insect host and arboviruses remains unclear [31].

Although the mechanism of how Wolbachia inhibits arboviruses is not fully known, Wolbachia density is correlated with viral interference in both native Drosophila [32] and transinfected St. aegypti [20]. In general, resident Wolbachia strains in wild populations do not appear to grow to such high densities as transinfected strains and therefore have less impact on arboviral vector competence. For example, a high-density $w$ Pip strain in a laboratory colonized line of $C x$. quinquefasciatus was observed to show resistance to $\mathrm{WNV}$, compared to a $w$ Pip cleared line [33]. However, lower density $w$ Pip infections found in field-collected $C x$. quinquefasciatus and $C x$. pipiens mosquitoes do not appear to be capable of inhibiting WNV infection and transmission [34]. Recent studies have shown that the removal of resident Wolbachia strains from St. albopicta, followed by transinfection of the Drosophila $w$ Mel strain, results in strong inhibition of both DENV and CHIKV [35, 36]. Therefore, the transinfection of mosquito species with Drosophila Wolbachia strains would likely provide strong inhibitory effects on arbovirus transmission.

\section{Resident Wolbachia Strains and Mosquito Population Invasion}

The potential impact of resident Wolbachia strains on the ability of introduced transinfected strains must be considered if species such as Cx. quinquefasciatus and St. albopicta are considered targets for Wolbachia biocontrol. The first point to consider is whether the resident strain actually inhibits the ability to form stable transinfected lines with Drosophila Wolbachia strains. Wolbachia bacteria are maternally inherited so are found at high densities in the reproductive tissues (ovaries for females). As Wolbachia transinfection involves the injection of preblastoderm embryos for the infection of the pole cells (that form the germline), the presence of high-density resident Wolbachia strains is likely to decrease the chances of success. However, an artificial triple Wolbachia infection in St. albopicta was successfully created with the $w$ Ri strain of Wolbachia from Drosophila simulans and yielded a new pattern of cytoplasmic incompatibility [37]. This generation of a 'superinfected' line with a resident strain and a transinfected strain has not been possible so far in $C x$. quinquefasciatus (despite significant efforts).

There may be species-specific differences between Wolbachia-mosquito host interactions that impact transinfection success. A stable, Wolbachia superinfected line with new transinfected strains ( $w \mathrm{Mel}$ and $w \mathrm{AlbB}$ ) was recently generated in St. aegypti (Joubert et al. unpublished data). The transinfection options for target mosquito species such as $C x$. quinquefasciatus that contains a single resident strain (wPip) include removal of the resident strain through antibiotic treatment or attempting to create a resident strain/ transinfected strain superinfection. If a single infection containing a novel transinfected Wolbachia strain (eg. w Mel) was generated, the crossing patterns induced between infected mosquitoes could still result in the invasion of the transinfected strain. Matings involving two different Wolbachia strains in Cx. quinquefasciatus would result in bidirectional CI [38] and two strains cannot stably co-exist in a given mosquito population. The strain that reaches the highest local frequency would likely reach fixation given that females infected with this strain would be at a reproductive advantage (more males to mate with that are compatible). However, if significant fitness costs are associated with a transinfected Wolbachia strain this would prevent the invasion of the population and replacement of the resident $w$ Pip strain.

The potential role of Wolbachia strains in the speciation and genetic evolution of mosquito populations over time through unidirectional or bidirectional CI patterns between different native or introduced strains should also be considered, in addition, to the resultant phenotypic effects [39]. Other forms of reproductive interference can also affect mosquito vector population dynamics and could therefore potentially impact on the spread of Wolbachia. For example, 
satyrization is a form of reproductive interference whereby males of one species mate with females of another, producing no viable offspring but leading to the mated female no longer being receptive to further insemination for the remaining life of that mosquito [40, 41]. This phenomenon is believed to result from male accessory gland products and has been linked to population displacement in certain areas of St. aegypti by St. albopicta [42]. As St. albopicta has a resident Wolbachia strain and St. aegypti does not, if CI of Wolbachia in St. albopicta is not itself involved in this mechanism, satyrization could potentially assist in or lead to the amplification of the driving force of Wolbachia into certain populations in a population replacement strategy.

In addition to the presence of resident Wolbachia strains, the presence of other microorganisms within the mosquito population is another important factor to consider, which could affect the introduction of any transinfected Wolbachia strain into that population. For example, the bacteria Asaia is stably associated with many mosquito species [43] and has been found to compete with Wolbachia within a host, seemingly preventing or reducing Wolbachia establishment. It has also been suggested that the presence of Asaia in certain mosquito species could be a reason for the absence of native Wolbachia strains in such species [44].

\section{Conclusion}

Wolbachia-based biocontrol strategies are likely to provide an environmentally benign and effective long-term control option for arboviral diseases such as dengue. Invasion and maintenance of transinfected Wolbachia strains in natural mosquito populations is likely to be cost effective given this should be self-sustaining through the CI phenotype and maternal transmission. Although significant advancements have been made in the implementation of Wolbachia for dengue biocontrol, the optimal Wolbachia strain (or combination of strains) to balance the inhibitory effects of DENV and fitness costs to the mosquito is still to be determined. A major area of research that needs addressing is how Wolbachia inhibits arboviruses in mosquitoes as further knowledge would allow more accurate predictions of the evolutionary consequences of strong selection pressure on DENV. This would likely provide better estimates of the effect Wolbachia would have on dengue transmission. Wolbachia's broad protective inhibition of human arboviruses suggests the potential of this strategy to reduce the transmission of other arboviral diseases. The presence of resident Wolbachia strains in some mosquito vectors must be considered if transinfection (with subsequent favourable phenotypic effects) is to be successfully implemented. If promising initial laboratory research and field trials can translate into effective biocontrol programs, Wolbachia may provide a novel method to control additional mosquito-borne diseases.

\section{Compliance with Ethical Standards}

Conflict of Interest The authors declare that they have no competing interests.

Human and Animal Rights and Informed Consent This article does not contain any studies with human or animal subjects performed by any of the authors.

Open Access This article is distributed under the terms of the Creative Commons Attribution 4.0 International License (http:// creativecommons.org/licenses/by/4.0/), which permits unrestricted use, distribution, and reproduction in any medium, provided you give appropriate credit to the original author(s) and the source, provide a link to the Creative Commons license, and indicate if changes were made.

\section{References}

Papers of particular interest, published recently, have been highlighted as:

- Of importance

1. Bhatt S, Gething PW, Brady OJ, Messina JP, Farlow AW, Moyes $\mathrm{CL}$, et al. The global distribution and burden of dengue. Nature. 2013;496:504-7. A recent review of worldwide dengue transmission and resulting disease.

2. Guzman MG, Halstead SB, Artsob H, Buchy P, Farrar J, Gubler DJ, et al. Dengue: a continuing global threat. Nat Rev Microbiol. 2010;8:S7-16.

3. Kilpatrick AM, Randolph SE. Drivers, dynamics, and control of emerging vector-borne zoonotic diseases. Lancet. 2012;380: 1946-55.

4. Iturbe-Ormaetxe I, Walker T, ONeill SL. Wolbachia and the biological control of mosquito-borne disease. EMBO Rep. 2011;12:508-18.

5. Werren JH, Baldo L, Clark ME. Wolbachia: master manipulators of invertebrate biology. Nat Rev Microbiol. 2008;6:741-51.

6. Hertig M, Wolbach SB. Studies on rickettsia-like micro-organisms in insects. J Med Res. 1924;44:329-374.7.

7. Hilgenboecker K, Hammerstein P, Schlattmann P, Telschow A, Werren JH. How many species are infected with Wolbachia?-a statistical analysis of current data. FEMS Microbiol Lett. 2008;281: 215-20.

8. Osei-Poku J, Han C, Mbogo CM, Jiggins FM. Identification of Wolbachia strains in mosquito disease vectors. PLoS ONE. 2012;7, e49922.

9. Baton LA, Pacidonio EC, Goncalves DS, Moreira LA. wFlu: characterization and evaluation of a native Wolbachia from the mosquito Aedes fluviatilis as a potential vector control agent. PLoS ONE. 2013;8, e59619.

10. Andrews ES, Xu G, Rich SM. Microbial communities within fieldcollected Culiseta melanura and Coquillettidia perturbans. Med Vet Entomol. 2014;28:125-32.

11. de Oliveira CD, Goncalves DS, Baton LA, Shimabukuro PH, Carvalho FD, and Moreira LA. Broader prevalence of Wolbachia in insects including potential human disease vectors. Bull Entomol Res. 2015:1-11.

12. Sinkins SP, Braig HR, O’Neill SL. Wolbachia superinfections and the expression of cytoplasmic incompatibility. Proc Biol Sci. 1995;261:325-30. 
13. Laven H. Eradication of Culex pipiens fatigans through cytoplasmic incompatibility. Nature. 1967;216:383-4.

14. Min KT, Benzer S. Wolbachia, normally a symbiont of Drosophila, can be virulent, causing degeneration and early death. Proc Natl Acad Sci U S A. 1997;94:10792-6.

15. Teixeira L, Ferreira A, Ashburner M. The bacterial symbiont Wolbachia induces resistance to RNA viral infections in Drosophila melanogaster. PLoS Biol. 2008;6, e2.

16. Hedges LM, Brownlie JC, O'Neill SL, Johnson KN. Wolbachia and virus protection in insects. Science. 2008;322:702.

17. Osborne SE, Leong YS, O'Neill SL, Johnson KN. Variation in antiviral protection mediated by different Wolbachia strains in Drosophila simulans. PLoS Pathog. 2009;5, e1000656.

18. Xi Z, Khoo CC, Dobson SL. Wolbachia establishment and invasion in an Aedes aegypti laboratory population. Science. 2005;310: 326-8.

19. McMeniman CJ, Lane RV, Cass BN, Fong AW, Sidhu M, Wang YF, et al. Stable introduction of a life-shortening Wolbachia infection into the mosquito Aedes aegypti. Science. 2009;323:141-4.

20. Walker T, Johnson PH, Moreira LA, Iturbe-Ormaetxe I, Frentiu FD, McMeniman CJ, et al. The wMel Wolbachia strain blocks dengue and invades caged Aedes aegypti populations. Nature. 2011;476: 450-3.

21. Moreira LA, Iturbe-Ormaetxe I, Jeffery JA, Lu G, Pyke AT, Hedges LM, et al. A Wolbachia symbiont in Aedes aegypti limits infection with dengue, Chikungunya, and Plasmodium. Cell. 2009;139: 1268-78.

22. Marinotti O, de Brito M, Moreira CK. Apyrase and alpha-glucosidase in the salivary glands of Aedes albopictus. Comp Biochem Physiol B: Biochem Mol Biol. 1996;113:675-9.

23. Hoffmann AA, Montgomery BL, Popovici J, Iturbe-Ormaetxe I, Johnson PH, Muzzi F, et al. Successful establishment of Wolbachia in Aedes populations to suppress dengue transmission. Nature. 2011;476:454-7.

24. Frentiu FD, Zakir T, Walker T, Popovici J, Pyke AT, van den Hurk A, et al. Limited dengue virus replication in field-collected Aedes aegypti mosquitoes infected with Wolbachia. PLoS Negl Trop Dis. 2014;8, e2688. Research showing strong dengue inhibition in wild Wolbachia-infected Stegomyia aegypti mosquitoes.

25. Ferguson NM, Kien DT, Clapham H, Aguas R, Trung VT, Chau $\mathrm{TN}$, et al. Modeling the impact on virus transmission of Wolbachiamediated blocking of dengue virus infection of Aedes aegypti. Sci Transl Med. 2015;7:279ra-37.

26. Lambrechts L, Ferguson NM, Harris E, Holmes EC, McGraw EA, O'Neill SL, Ooi EE, Ritchie SA, Ryan PA, Scott TW, Simmons CP, and Weaver SC. Assessing the epidemiological effect of Wolbachia for dengue control. Lancet Infect Dis. 2015. Paper highlights a practical approach for dengue reduction through field release of Wolbachia-infected mosquitoes.

27. van den Hurk AF, Hall-Mendelin S, Pyke AT, Frentiu FD, McElroy $\mathrm{K}$, Day A, et al. Impact of Wolbachia on infection with chikungunya and yellow fever viruses in the mosquito vector Aedes aegypti. PLoS Negl Trop Dis. 2012;6, e1892.

28. Jeffries CL, Walker T. The potential use of Wolbachia-based mosquito biocontrol strategies for Japanese encephalitis. PLoS Negl Trop Dis. 2015;9, e0003576.

29. Sinkins SP, Braig HR, O’Neill SL. Wolbachia pipientis: bacterial density and unidirectional cytoplasmic incompatibility between infected populations of Aedes albopictus. Exp Parasitol. 1995;81: 284-91.

30. Goddard LB, Roth AE, Reisen WK, Scott TW. Vector competence of California mosquitoes for West Nile virus. Emerg Infect Dis. 2002;8:1385-91.

31. Zug R, Hammerstein P. Bad guys turned nice? A critical assessment of Wolbachia mutualisms in arthropod hosts. Biol Rev Camb Philos Soc. 2015;90:89-111.

32. Osborne SE, Iturbe-Ormaetxe I, Brownlie JC, O'Neill SL, Johnson KN. Antiviral protection and the importance of Wolbachia density and tissue tropism in Drosophila simulans. Appl Environ Microbiol. 2012;78:6922-9.

33. Glaser RL, Meola MA. The native Wolbachia endosymbionts of Drosophila melanogaster and Culex quinquefasciatus increase host resistance to West Nile virus infection. PLoS ONE. 2010;5, e11977.

34. Micieli MV, Glaser RL. Somatic Wolbachia (Rickettsiales: Rickettsiaceae) levels in Culex quinquefasciatus and Culex pipiens (Diptera: Culicidae) and resistance to West Nile virus infection. J Med Entomol. 2014;51:189-99.

35. Blagrove MS, Arias-Goeta C, Failloux AB, Sinkins SP. Wolbachia strain wMel induces cytoplasmic incompatibility and blocks dengue transmission in Aedes albopictus. Proc Natl Acad Sci U S A. 2012;109:255-60.

36. Blagrove MS, Arias-Goeta C, Di Genua C, Failloux AB, Sinkins SP. A Wolbachia wMel transinfection in Aedes albopictus is not detrimental to host fitness and inhibits Chikungunya virus. PLoS Negl Trop Dis. 2013;7, e2152.

37. Fu Y, Gavotte L, Mercer DR, Dobson SL. Artificial triple Wolbachia infection in Aedes albopictus yields a new pattern of unidirectional cytoplasmic incompatibility. Appl Environ Microbiol. 2010;76: 5887-91.

38. Sinkins SP, Walker T, Lynd AR, Steven AR, Makepeace BL, Godfray HC, et al. Wolbachia variability and host effects on crossing type in Culex mosquitoes. Nature. 2005;436:257-60.

39. Atyame CM, Labbe P, Rousset F, Beji M, Makoundou P, Duron O, et al. Stable coexistence of incompatible Wolbachia along a narrow contact zone in mosquito field populations. Mol Ecol. 2015;24: 508-21.

40. Craig Jr GB. Mosquitoes: female monogamy induced by male accessory gland substance. Science. 1967;156:1499-501.

41. Tripet F, Lounibos LP, Robbins D, Moran J, Nishimura N, Blosser EM. Competitive reduction by satyrization? Evidence for interspecific mating in nature and asymmetric reproductive competition between invasive mosquito vectors. Am J Trop Med Hyg. 2011;85:265-70.

42. Bargielowski IE, Lounibos LP, Carrasquilla MC. Evolution of resistance to satyrization through reproductive character displacement in populations of invasive dengue vectors. Proc Natl Acad Sci U S A. 2013;110:2888-92.

43. Favia G, Ricci I, Damiani C, Raddadi N, Crotti E, Marzorati M, et al. Bacteria of the genus Asaia stably associate with Anopheles stephensi, an Asian malarial mosquito vector. Proc Natl Acad Sci U S A. 2007;104:9047-51.

44. Rossi P, Ricci I, Cappelli A, Damiani C, Ulissi U, Mancini MV, et al. Mutual exclusion of Asaia and Wolbachia in the reproductive organs of mosquito vectors. Parasit Vectors. 2015;8:278. 